\title{
Fire Detection Using Multi Criteria Image Processing Technique in Video Sequences
}

\author{
Behrouz Memarzadeh*1, Mohammad Ali Mohammadi ${ }^{2}$ \\ ${ }^{1}$ Departement of Electrical and Computer Engineering, Bahonar University \\ ${ }^{2}$ Departement of Electrical Engineering, Bahonar University \\ *Corresponding author, e-mail: b.memarzadeh@eng.uk.ac.ir
}

\begin{abstract}
Vision-based flame detection has drawn significant attention in the past decade with camera surveillance systems becoming ubiquitous. This paper proposes a multi criterion method to detect fire or flames by processing the video data generated by a high speed camera. Since flame images are special class of images, some of the unique features of a flame may be used to identify flame. There are some differences between flame images and other general images. By using these features we are able to detect fire correctly with least false alarm. In this paper we present an algorithm which can detect fire and reduce number of false alarms by counting number of identified pixels. In the algorithm, we preprocess the images to have better results. So first we adjust the gray level of a flame image according to its statistical distribution to have better processing. After that we try to extract fire features in images. First by using color characteristics, the ratio of red to green, we can identify probable fire-like or fire like pixels. Second, to highlight the regions with high gray level contrast at their edges, we use the extended prewitt filter. We use AND operation on two above processing images to remove unrelated pixels, at last by using flicker frequency, the oscillating change in the number of identified pixels over time is transformed into the frequency domain to complete detection algorithm. Simulation proves the algorithm ability to detect fire in different situations in video sequences.
\end{abstract}

Keywords: fire detection, color classification, high pass filter, fast fourier transform

Copyright $\odot 2015$ Institute of Advanced Engineering and Science. All rights reserved.

\section{Introduction}

In recent years, fire detection based on systems that use computer vision techniques has been widely researched because of the relative advantages of computer vision over conventional sensors, such as infrared, optical, or ion sensors, which depend on certain characteristics of fire [1-3]. Today's deflagration detection systems primarily focus on the fast identification of the hazard. This means deflagrations must be detected in their initial stage before they unfold their endangering potential. Consequently, the sooner a deflagration is detected the higher are the chances to suppress the following effects such as fast increasing pressure and high temperatures. For a good detection, a visual based approach for deflagration detection is a serious alternative to the conventional photodiode-based systems. The use of image processing techniques for fire detection has already been a well established method for at least two decades [4]. Most of the fire detection approaches are mainly based on the typical fire color characteristics and the motion of the fire or the flame [5]. Phillips et al. [6] used color and temporal variation as clues. Whether a pixel belongs to fire area depends on its color and significant temporal variation. Giuseppe Marbach et al. [7] used temporal variation of fire intensity to capture candidate regions and extract characteristic color features to detect fire. Toreyin et al [8] developed a wavelet method for detecting whether a pixel is fire or not. They keep on tracking the history of red channel for each pixel which is part of fire contour in a relative short time, and take them as the input of wavelet method. As it is presented in Hamins et al [9], fire or flames pulsate in a special frequency range, called flicker frequency $(1-10 \mathrm{~Hz})$. Fire detection approaches based on this knowledge transform the temporal variation of a fire shape into a frequency spectrum by using wavelet [10] or Fourier transform [11]. If the resulting frequencies are in the flicker range, the pixel can be confirmed as a fire or flame. The method presented in this paper uses a multi criterion image processing technique which includes 4 steps. In section 1 we preprocess the image by histogram equalization to have better final 
results. In section2, by defining the ratio of $R / G$, we try to neglect some pixels. Since fire release a huge amount of energy in a short time, we can use a high pass filter to find edges in an image, so an extended prewitt filter is presented in section3. The analysis of the frequency spectrum facilitates identifying fires by their typical flicker frequency. So in section4 we use fast fourier transform to find whether the selected pixels found in previous steps are related to a firelike process or not.

\section{Preprocessing Step}

\subsection{Histogram Equalization}

Before we start the fire detection algorithm, we preprocess the images to have better result. Sometimes the fire data is bluer and the quality of the image is not good. So before implementing the algorithm, we adjust the gray level of a flame image according to its statistical distribution. Considering a discrete grayscale image $\mathrm{x}$ and letting $n_{i}$ be the number of occurrences of gray level of $i$, the probability of the occurrence of a pixel of gray level $i$ in the image is [12].

$$
P_{x}(\mathrm{i})=\frac{n_{i}}{n}, \quad 0 \leq i \leq L
$$

Where $L$ is the total number of gray levels in the image, $n$ the total number of pixels in the image, and $P_{x}(\mathrm{i})$ the histogram for pixels with $\mathrm{i}$, normalized to $[0,1]$. Also, the cumulative distribution function (CDF) corresponding to $P_{x}$ can be defined as: $C D F_{x}(\mathrm{i})=\sum_{j=0}^{i} P_{x}(\mathrm{j}) \leq 1$. Which is also the accumulated normalized histogram of the image. Next, create a transformation of form $y=T(x)$ to produce a new image $\{y\}$, such that its CDF will be linearized across the value range with a constan number $\mathrm{K}, C D F_{y}(\mathrm{i})=i K$. To map the values back to their original range, the following transformation is applied to the result: $y^{\prime}=y \times(\max \{\mathrm{x}\}-\min \{\mathrm{x}\})+\min \{\mathrm{x}\}$. The best histogram equalization is when the histogram curve is linear but since image data is discrete, it is not possible. As shown in Figure $1(a)$, the more histogram curve is closer to linear curve, the better image quality we have.

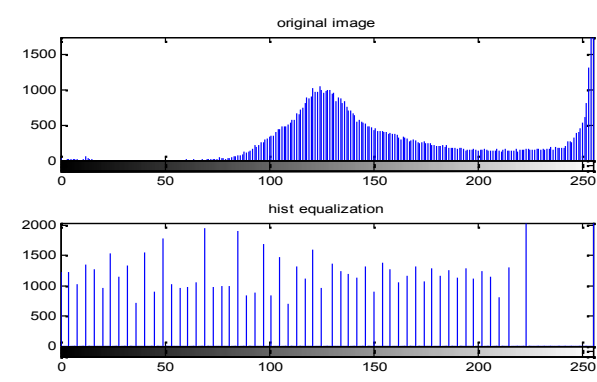

Figure 1(a). Histogram curves of Original image and Equalized image
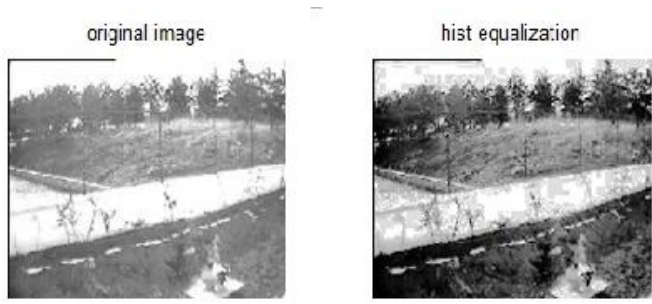

Figure1(b). Original image and Equalized image

In fact, When we supply a desired histogram, histogram chooses the grayscale transformation $\mathrm{T}$ to minimize $\left|c_{1}(\mathrm{~T}(\mathrm{k}))-\mathrm{c}_{0}(\mathrm{k})\right|$ where $\mathrm{c} 0$ is the cumulative histogram of $\mathrm{A}, \mathrm{c} 1$ is the cumulative sum of histogram for all intensities $\mathrm{k}$. 


\section{Detection Algorithm}

\subsection{Color Extraction for Candidate Fire-Like Pixels}

Identification of each potential deflagration-like or fire-like pixel is a vital process for every detection algorithm. The detection algorithm must focus on typical features of the combustion processes, which need to be detected. The chromatic features of a deflagration are already presented in Schroeder et al [13]. Color space feature is used in [14] to identify fire-like pixels. If $R \geq G \geq B$ and $R \geq R_{\text {th }}$ then the pixel can belong to fire ( $R_{\text {th }}$ is defined as threshold value). So the color pixel is still an important feature of a fire and in our algorithm the ratio of red to green is considered as a vital characteristic of a fire and if the ratio value is upper than a predefined threshold value we consider it as a fire-like pixel. For the reason that the fire color feature in different scenarios would be affected by the environmental luminance, thus to effectively detect fires under these circumstances, fire color criteria should be proposed. Another advantage of using color space is that we eliminate unwanted data and reduce data to have a faster processing for on-line detection. This section explores the fire color in RGB color space. In [15] the author mentioned that in a common fire scenario the value of $R$ is greater than $G$ and $B$. In the first step of detection algorithm we concentrate on the ratio of red to green pixels $(R / G)$ to separate fire-like pixels from non-fire pixels. By defining a predefined threshold for the ratio, we consider the values greater than threshold as a candidate for fire pixels.

$$
\frac{R(\mathrm{x}, \mathrm{y})}{G(\mathrm{x}, \mathrm{y})} \geq t h 1 \quad \longrightarrow \quad \text { candidate fire pixel }
$$

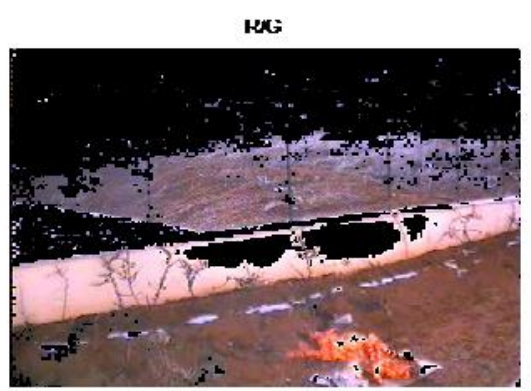

Figure 2. The ratio of $\mathrm{R} / \mathrm{G}$

As shown in Figure 2, in RGB color space, the values lower than predefined threshold(th1) is considered as zero, and greater than predefined threshold is considered as a candidate for fire pixels, however it may not be fire as can be seen. Then this image is converted to gray scale for AND operation in the next steps.

\subsection{Using the Extended of Prewitt Operator for Finding Basic Edges}

Another important feature of a fire-like pixel is the high variation in intensity due to the emission of a huge quantity of energy in a very short time [16]. Here we identify pixels with a fast variation in intensity. To have a successful detection, the high- dynamic parts of the pixel intensity must be identified. So using a high pass filter is a proper way to candidate some other pixels rather than identified pixels in previous section. We use extended prewitt operator to find basic edges to have final better result in detection algorithm. Finding basic edges is achieved by finding the gradients of all the pixels in the image so as to highlight the regions with high gray level contrast at their edges. The algorithm then tracks the edge along these regions and suppresses any pixels that are not at the peaks of the gradients. If the magnitude of the gradient is above high threshold ${ }^{t h} h_{2}$ it is deemed an edge. Since prewitt operator is a horizontal edge detector we use extended operator to find edges in both vertical and horizontal pixels to have better edge detection in all directions. By using extended prewitt operator we can have edge 
detection in all directions. Mathematically, the edges are represented by first and second order derivatives. The first order derivative of a $2 D f(x, y)$ is defined as:

$$
\nabla f=\left[\begin{array}{l}
g x \\
g y
\end{array}\right]=\left[\begin{array}{l}
\frac{\partial y}{\partial x} \\
\frac{\partial y}{\partial x}
\end{array}\right]
$$

This vector has the important geometrical properties that it points in the direction of the greatest rate of change of $f$ at location $(x, y)$. The prewitt operator is expressed as follows.

The magnitude of vector $\nabla f$ is:

$$
M(\mathrm{x}, \mathrm{y})=\operatorname{mag}(\nabla \mathrm{f})=\sqrt{G_{x}{ }^{2}+G_{y}}{ }^{2}
$$

is the value of the rate of change in the direction of the gradient vector note that gx, gy and $M(x, y)$ are images of the same size as the original, created when $x$ and $y$ are allowed to vary over all pixel locations in $\mathrm{f}$. It is a common practice to refer image as the gradient image or simply as the gradient when the magnitude is clear. Another important quantity is the direction of the gradient vector is:

$$
\alpha(\mathrm{x}, \mathrm{y})=\operatorname{tg}^{-1}\left(\frac{g y}{g x}\right)
$$

$\boldsymbol{\alpha}(\mathrm{x}, \mathrm{y})$ also is an image of the same size as the original created by the array division of image gy by image gx. The direction of an edge at an arbitrary point $(x, y)$ is orthogonal to the direction $\boldsymbol{\alpha}(\mathrm{x}, \mathrm{y})$ of the gradient vector at the point. For defining the edges in all directions we use an extended prewitt filter as below:

$$
G_{p}=\left[\begin{array}{llll}
1 & 1 & 1 & \\
0 & 0 & 0 & \\
-1 & -1 & -1
\end{array}\right] \quad G_{p}^{\prime}=\left[\begin{array}{ccc}
1 & 0 & -1 \\
1 & 0 & -1 \\
1 & 0 & -1
\end{array}\right]
$$

And the extended operator we used is:

$$
G=\sqrt{G_{P}{ }^{2}+G_{P}^{\prime}}{ }^{2}
$$

The result of extended prewitt operation is shown in Figure 2. The pixels with high intensity variation, including fire pixels, are clear in Figure 2.

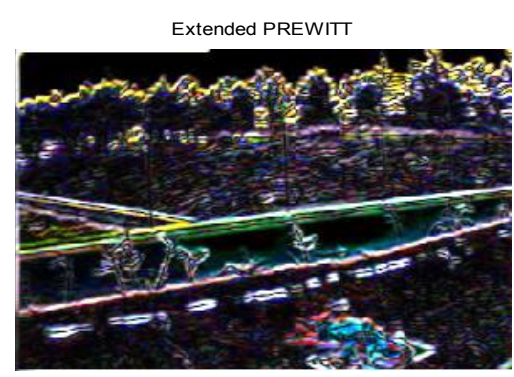

Figure 3. Edge detection by extended prewitt operator 


\subsection{AND Operator}

In [17], by knowing fire features and after extracting fire features based on these knowledge, the author uses pixel-by-pixel AND operation to excluded some non- flame areas and classify the remaining areas as candidate flame regions, but the problem is, we need the background image of the region for detecting fire, so any changes in the environment can result in changing the background image and readjusting the algorithm. Here we use the AND operation to exclude the non-fire pixels which are considered as candidate fire pixels in the two previous sections. Here, a pixel-by-pixel AND operation is performed with the two previous images for eliminating unwanted pixels.

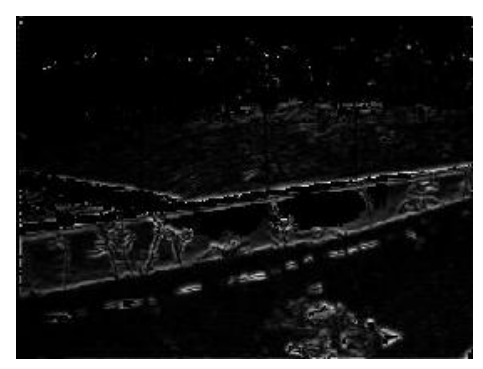

Figure 3. AND operation of two previous images

As it can be seen in Figure 3, by using AND operation we can eliminate many pixels in the two previous steps which has been considered as fire, as well as remaining the real fire pixels. Yet, there are some unwanted pixels that don't belong to fire which we must remove them. For this purpose we can use Fast Fourier Transformation (FFT) in next step.

\subsection{Frequency-Based Fire Detection}

In parallel to the deflagration verification, it is possible to check whether the selected pixels(after AND step) are related to a fire-like process. This fire classification mechanism is based on the knowledge of the typical flicker frequency range of fires and flames [18]. Here, we analyze the fire part-like of the frequency spectrum by transforming the time-depending number of identified pixels in previous step (AND operation) into the frequency domain. We can calculate the contour fluctuation to decide whether the region is fire or not, due to the shape of fire that changes every frame. We assume that the boundary has $\mathrm{N}$ points which are expressed in complex form:

$$
\left\{\mathrm{z}_{i} \mid z_{i}=x_{i}+j y_{i}\right\}
$$

Where $\left(x_{i}, y_{j}\right)$ are the coordinates of the ith point on the boundary. In order to detect flicker or oscillations in pixels due to fire in a reliable manner, the video capture rate should be high enough to capture high-frequency flicker in flames. We chose time-efficient evaluation fast Fourier transform (FFT) with a maximum length of 1024 data points. Based on the NyquistShannon sampling theorem, the maximum frequency $f_{\max }$ is half the sampling frequency $(210 \mathrm{~Hz})$. The presence of the characteristic frequencies within the normalized frequency range from 1 to $9 \mathrm{~Hz}$ is determined by:

$$
a=\frac{\sqrt{\sum_{f=1}^{9 H z}|X(\mathrm{f})|^{2}}}{\sqrt{\sum_{f=1}^{f_{\max }}|X(\mathrm{f})|^{2}}} \quad a \in[0,1]
$$

Where 'a' denotes the power ratio of the frequency band from 2 to $8 \mathrm{~Hz}$ and $X(f)$ represents the amplitude value at the frequency $f$. The sequence contains, depending on the desired frequency resolution, the number of identified pixels of 512 or 1024 images. By using a high speed 
camera, capturing $210 \mathrm{frames} / \mathrm{s}$, the detection time is $4.8 \mathrm{~s}$. Typical fires or flames show a ratio a in the range of 0.62 to 0.91 , so in simulation we consider these values as fire. After all these verification steps the last result for detection in Figure 4. It can be seen that the detection algorithm has a good ability to eliminate noise from fire.

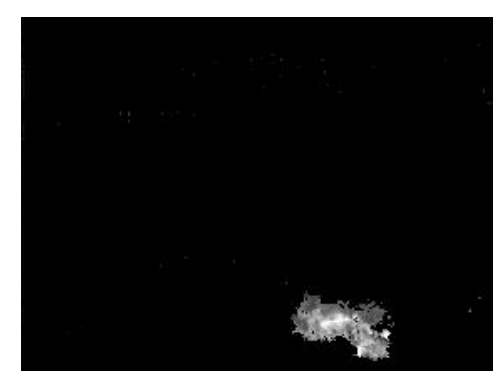

Figure 5. Final fire detection

\section{Disturbing Sources}

Disturbing sources (false alarms) are a major challenge for the detection algorithm. Arc welding or flames of a cigarette or lighter are potential non- hazardous events. Finally the number of identified pixels is counted to distinguish between real fire and sources like candles or sources which cause false alarm. If the counted pixels are above a predefined threshold (th3) the alarm is sent to sprinkler systems for activation, otherwise alarm signal doesn't send and sprinkler systems won't be activated. Briefly, the flowchart of fire detection algorithm is shown below:

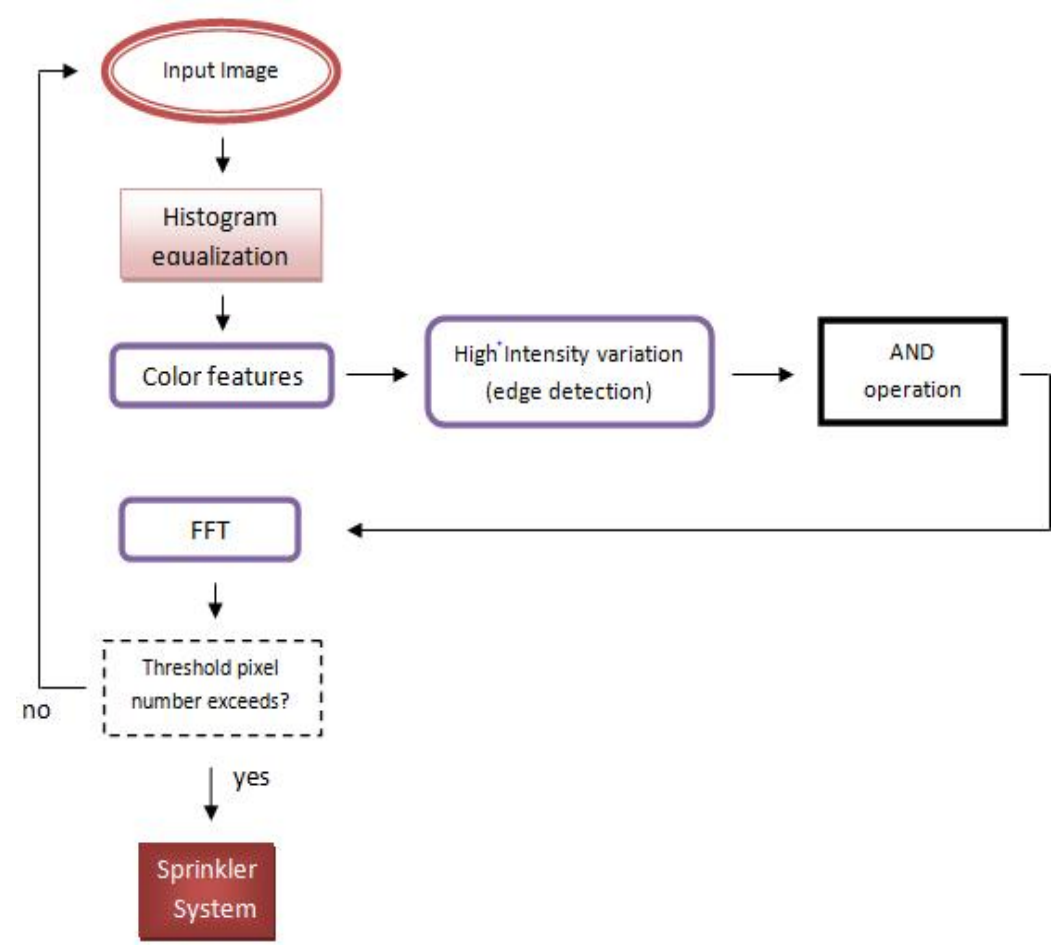

Fire Detection Using Multi Criteria Image Processing Technique in... (Behrouz Memarzadeh) 

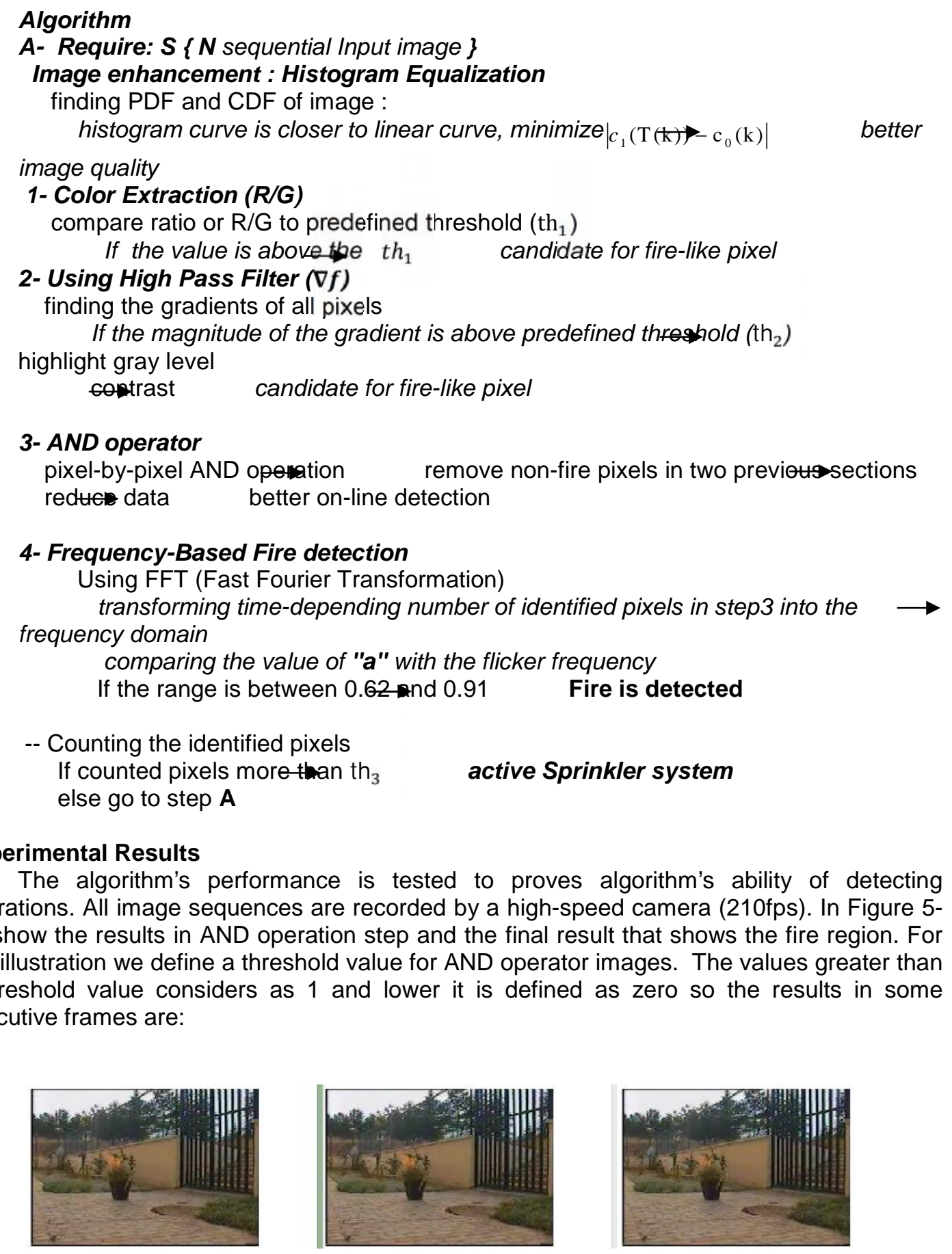

Figure 5. Original image
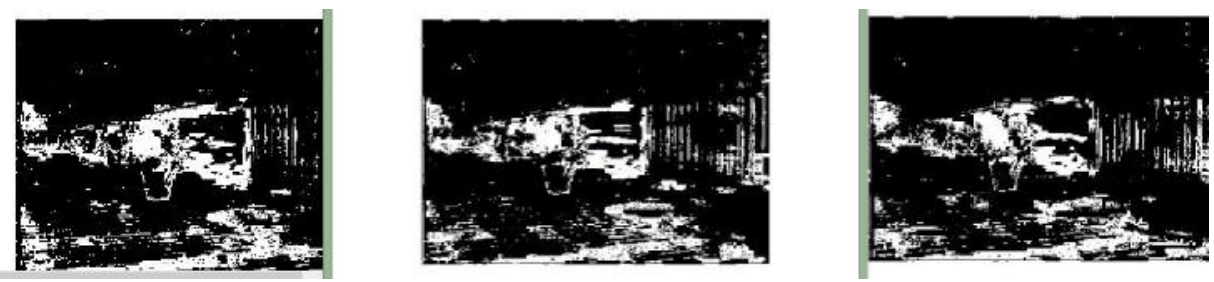

Figure 6. AND operation with predefined threshold

TELKOMNIKA Vol. 16, No. 1, October 2015 : $136-144$ 

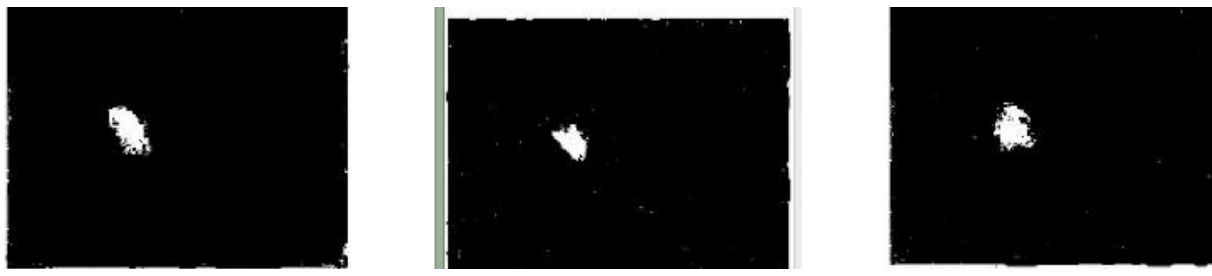

Figure 7. Final fire detection

We define the number of identified pixels in both video sequences $t$ reduce false alarms. In Figure 8-9 the number of identified pixels are shown:

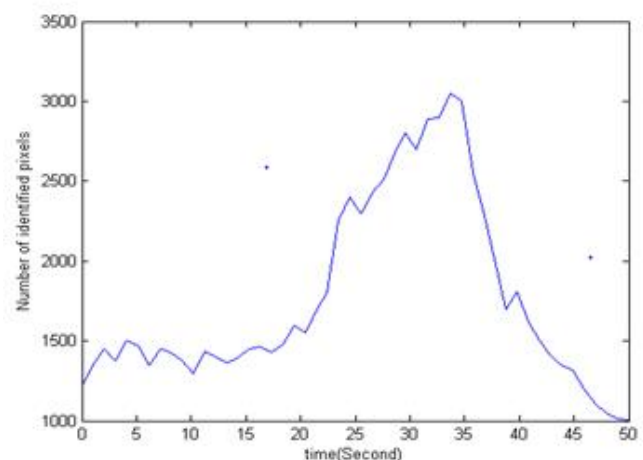

Figure7-a Number of identified pixels for the first fire

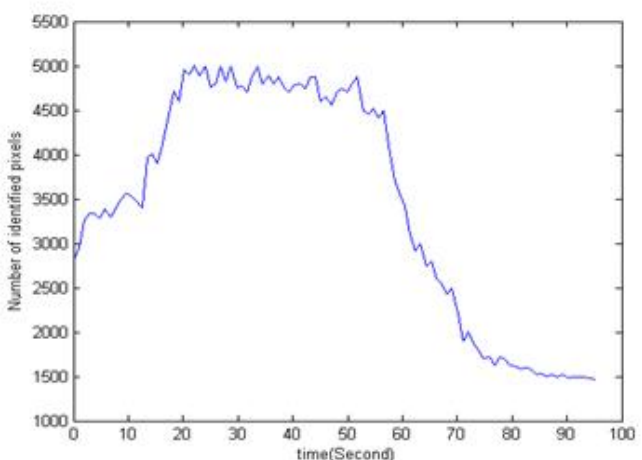

Figure7-b Number of identified pixels for the second fire situation

By defining a threshold value(th4), we consider it fire if the counted pixels are more than predefined value, else it is not fire. However it absolutely depends on the distance between fire and camera. So the predefined value can be set by experimental computation after installing camera and doing some experiments. As it is obvious,the predefined threshold for both situation is diffrent. For the first situation between 20-60s fire is growing and it is considered as a hazardous fire, so sprinkler systems must work on their peak operation.

\section{Conclusion}

Reliable deflagration detection improves safety in very sensitive environments such as military crew compartments as well as production lines using explosive materials, which require very detection times.

the detection algorithm based on one camera system is not able to distinguish between a deflagration or a flashing lighter directly in front of the camera. Both events appear as a hazardous process, because the algorithm is not capable of a scaled evaluation of the identified event. This problem can be solved by expanding the number of cameras. Accordingly, more than one camera system in combination with the algorithm facilitates three-dimensional detection by determining the location and size of the event. If this happen we are able to active the sprinkler systems near the fire instead of activating all sprinkler system to suppress it in an economic way. Here by using a multi-criterion technique we are able to detect fire from sources like sunlight, a moving object in both close and outdoor spaces and counting of identified pixels enable us to differentiate fire from sources such as cigarette or arc welding.

\section{References}

[1] BU Töreyin, Y Dedeoglu, U Gudukbay, AE Cetin. Computer vision based method for real-time fire and flame detection. PatternRecognit.Lett.27. 2006: 49-58. 
[2] BC Ko, SY Kwak. A survey of computer vision-based natural disaster warning systems. Opt.Eng. 2012; 51: 1-11.

[3] BC Ko, SJ Ham, JY Nam. Modeling and formalization of fuzzy finite automata for detection of irregular fire flames. IEEETrans.Circuits Syst. Video Technol. 2011; 21: 1903-1912.

[4] G Healey, D Slater, T Lin, B Drda, AD Goedeke. A system for real-time fire detection. Proceedings of the IEEE Conference on Computer Vision and Pattern Recognition. 1993: 605-606.

[5] S Verstockt, B Merci, B Sette, P Lambert, R Vande Walle. State of the art in vision-based fire and smoke detection. Proceedings of the 14th International Conferenceon Automatic Fire Detection. 2009. 2: 285-292.

[6] Phillips III W, Shah M, Lobo NV. Flame recognition in video. Pattern Recognition Letters 23. 2002: 319-327.

[7] Giuseppe Marbach, et al. An image processing technique for fire detection in video images. Fire Safety Journal 41. 2006: 285-289.

[8] B Ugur Toreyin, Yigithan Dedeoglu, Ugur Gudukbay, A Enis Cetin. Computer vision based method for real-time fire.

[9] A Hamins, JC Yang, T Kashiwagi. An experimental investigation of the pulsation frequency of flames. Symposium (International) on Combustion. 1992; 24(1): 1695-1702.

[10] BU Töreyin, Y Dedeoglu, U Güdükbay, AE Cetin. Computer vision based method forreal-time fire and flame detection. Pattern Recognit. Lett. 27. 2006: 49-58.

[11] CB Liu, N Ahuja. Vision based fire detection. Proceedings of the International Conference on Pattern Recognition. 2004; 4: 134-137.

[12] KR Castleman. Digital Image Processing. Englewood Cliffs, NJ: Prentice-Hall. 1995.

[13] T Schroeder, K Krueger, F Kuemmerlen. Fast detection of deflagrations using image processing. Proceedings of the Suppression, Detection and Signaling Research and Applications Symposium. 2013.

[14] Lu Song, et al. The research of real-time forest fire alarm algorithm based on video. Intelligent HumanMachine Systems and Cybernetics (IHMSC), 2014 Sixth International Conference on IEEE. 2014; 1.

[15] CHEN Juan, BAO Qifu. International Symposium on Safety Science and Technology Digital image processing based fire flame color and oscillation frequency analysis. 2012.

[16] Qiu Tian, Yong Yan, Gang Lu. An auto adaptive edge-detection algorithm for flame and fire image processing. Instrumentation and Measurement, IEEE Transactions. 2012; 61(5): 1486-1493.

[17] Han Dongil, Byoungmoo Lee. Flame and smoke detection method for early real-time detection of a tunnel fire. Fire Safety Journal. 2009; 44(7): 951-961.

[18] A Hamins, JC Yang, T Kashiwagi. An experimental investigation of the pulsation frequency of flames. Symposium (International) on Combustion. 1992; 24(1): 1695-1702. 